\title{
Correction to: Determining parameters of Moon's orbital and rotational motion from LLR observations using GRAIL and IERS-recommended models
}

\author{
Dmitry A. Pavlov ${ }^{1}$. James G. Williams ${ }^{2}$. Vladimir V. Suvorkin ${ }^{1}$
}

Published online: 2 December 2019

(c) Springer Nature B.V. 2019

\section{Correction to: Celest Mech Dyn Astr (2016) 126:61-88 https://doi.org/10.1007/s10569-016-9712-1}

Correction 1. Equation (15) must be

$$
\begin{aligned}
\frac{I}{m}= & \frac{2 R_{M}^{2} \tilde{J}_{2}}{2 \beta-\gamma+\beta \gamma}\left[\begin{array}{ccc}
1-\beta \gamma & 0 & 0 \\
0 & 1+\gamma & 0 \\
0 & 0 & 1+\beta
\end{array}\right]-\frac{I_{c}}{m} \\
& -k_{2} \frac{\mu_{E}}{\mu_{M}}\left(\frac{R_{M}}{r}\right)^{5}\left[\begin{array}{ccc}
x^{2}-\frac{1}{3} r^{2} & x y & x z \\
x y & y^{2}-\frac{1}{3} r^{2} & y z \\
x z & y z & z^{2}-\frac{1}{3} r^{2}
\end{array}\right] \\
& +k_{2} \frac{R_{M}^{5}}{3 \mu_{M}}\left[\begin{array}{cccc}
\omega_{x}^{2}-\frac{1}{3}\left(\omega^{2}-n^{2}\right) & \omega_{x} \omega_{y} & \omega_{x} \omega_{z} \\
\omega_{x} \omega_{y} & \omega_{y}^{2}-\frac{1}{3}\left(\omega^{2}-n^{2}\right) & \omega_{y} \omega_{z} \\
\omega_{x} \omega_{z} & \omega_{y} \omega_{z} & \omega_{z}^{2}-\frac{1}{3}\left(\omega^{2}+2 n^{2}\right)
\end{array}\right]
\end{aligned}
$$

In the original, the bottom-right diagonal element of the last term had $\left(\omega^{2}-n^{2}\right)$. Correction 2. Equation (24) must be

$$
\begin{aligned}
\boldsymbol{\Delta}_{\text {solidmoon }}= & \frac{\mu_{A} R_{M}^{4}}{\mu_{M} r_{\mathrm{MA}}^{3}}\left[\frac{h_{2}}{2}\left(3\left(\hat{\boldsymbol{r}}_{\mathrm{MA}} \cdot \hat{\boldsymbol{l}}\right)^{2}-1\right) \hat{\boldsymbol{l}}\right. \\
& \left.+3 l_{2}\left(\hat{\boldsymbol{r}}_{\mathrm{MA}} \cdot \hat{\boldsymbol{l}}\right)\left(\hat{\boldsymbol{r}}_{\mathrm{MA}}-\left(\hat{\boldsymbol{r}}_{\mathrm{MA}} \cdot \hat{\boldsymbol{l}}\right) \hat{\boldsymbol{l}}\right)\right]
\end{aligned}
$$

In the original, $\hat{r}_{\text {MA }}$ was given instead of $\hat{l}$ at the end of the first line before the plus.

The original article can be found online at https://doi.org/10.1007/s10569-016-9712-1.

Dmitry A. Pavlov dpavlov@iaaras.ru

1 Institute of Applied Astronomy RAS, Kutuzov embankment 10, St. Petersburg 191187, Russia

2 Jet Propulsion Laboratory, California Institute of Technology, Pasadena, CA 91109, USA 
Correction 3. In Table 8, lines 7-9, the first columns should be $\omega_{c} \cdot \mathrm{x}, \boldsymbol{\omega}_{c} \cdot \mathrm{y}, \boldsymbol{\omega}_{c} . \mathrm{z}$. In the original, there were dots above the omegas.

Publisher's Note Springer Nature remains neutral with regard to jurisdictional claims in published maps and institutional affiliations. 